\title{
ITS-90 Density of Water Formulation for Volumetric Standards Calibration
}

\section{Frank E. Jones}

32 Orchard Way South, Potomac, MD 20854

\section{and}

\section{Georgia L. Harris}

National Institute of Standards and Technology, Gaithersburg, MD 20899
A ncw formulation of the density of airsaturated water as a function of temperature on the 1990 International Temperature Scale (ITS-90) is presented. Also, a new equation for calculating isothermal compressibility as a function of temperature on ITS-90 was developed. The equations are to be used to calculate the density of water, in the temperature range 5 to $40^{\circ} \mathrm{C}$ on ITS-90, used in the gravimctric determination of the volume of volumetric standards.
Key words: air-saturatcd water; calibration; density of water; isothermal compressibility; ITS.90; volumetric standards.

Accepted: April 27, 1992

\section{Introduction}

In the gravimetric determination of the volume (calibration) of volumetric standards, water is used as the calibrating fluid. The volume is calculated from the mass and density of the water. In many quarters, the formulation of Wagenbreth and Blanke [1] is used to calculate the density of water. In this paper, a new formulation of the density of water (based primarily on the work of Kell [2]) as a function of temperature on the 1990 International Temperature Scale is presented.

\section{Kell's Formulations}

\subsection{Density of Water}

In 1975, Kell [2] published a new formulation for the density of air-free water at a pressure of $101.325 \mathrm{kPa}$ ( 1 atmosphere) valid from 0 to $150^{\circ} \mathrm{C}$ "that is in improved agreement with most data sets." The Kell formulation is

$$
\begin{aligned}
& \rho\left(\mathrm{kg} \mathrm{m}^{-3}\right)=(999.83952+16.945176 t \\
& -7.9870401 \times 10^{-3} t^{2}-46.170461 \times 10^{-6} t^{3} \\
& \left.+105.56302 \times 10^{-9} t^{4}-280.54253 \times 10^{-12} t^{5}\right) \\
& \quad /\left(1+16.897850 \times 10^{-3} t\right.
\end{aligned}
$$

where $t$ is temperature in ${ }^{\circ} \mathrm{C}$ on the 1968 International Practical Temperature Scale (IPTS-68).

\subsection{Isothermal Compressibility}

Kell also developed equations for calculation of the isothermal compressibility, $\kappa_{T}$, of air-free water [2]. In the temperature range 0 to $100^{\circ} \mathrm{C}$ on IPTS68 , the equation can be expressed as

$$
\begin{gathered}
\kappa_{T}=\left(50.88496 \times 10^{-8}+6.163813 \times 10^{-9} t\right. \\
+1.459187 \times 10^{-11} t^{2}+20.08438 \times 10^{-14} t^{3} \\
\left.-58.47727 \times 10^{-17} t^{4}+410.4110 \times 10^{-20} t^{5}\right) \\
\quad /\left(1+19.67348 \times 10^{-3} t\right),
\end{gathered}
$$

where $\kappa_{T}$ is isothermal compressibility in $(\mathrm{kPa})^{-1}$. 


\section{New Formulations}

\subsection{Density of Air-Free Water}

In the present work, the Kell calculated values of $\rho$ were fitted over the temperature range 5 to $40^{\circ} \mathrm{C}$ on the new 1990 International Temperature Scale (ITS-90) [3] to an equation quartic in temperature. The equation is

$$
\begin{aligned}
& \rho\left(\mathrm{kg} \mathrm{m}^{-3}\right)=999.85308+6.32693 \times 10^{-2} t \\
& \quad-8.523829 \times 10^{-3} t^{2}+6.943248 \times 10^{-5} t^{3} \\
& \quad-3.821216 \times 10^{-7} t^{4} .
\end{aligned}
$$

In contrast with the Kell equation, a term in $t^{5}$ is not necessary due at least in part to the fact that the 0 to $4{ }^{\circ} \mathrm{C}$ region, in which $\rho$ increases with increasing temperature, has been excluded. Equation (3) applies to air-free water.

Values of the density of air-free water were calculated for temperatures (ITS-90, $t_{90}$ ) between 4.999 and $39.990^{\circ} \mathrm{C}$ using Eq. (3) and compared with corresponding Kell values. The estimate of the standard deviation (SD) of the difference was $0.00034 \mathrm{~kg} \mathrm{~m}^{-3}$. The ratio of SD to the mean value of density was $3.4 \times 10^{-7}$, which is negligible.

\subsection{Conversion of IPTS- 68 to ITS-90}

A very simple equation relating ITS-90 temperature, $t_{90}$, to IPTS- 68 temperature, $t_{68}$, has been used in the present work to generate values of $t_{90}$ for the development of Eq. (3). The equation for the temperature range 0 to $40^{\circ} \mathrm{C}$ is

$$
t_{90}=0.0002+0.99975 t_{68} \text {. }
$$

In the temperature range 0 to $100^{\circ} \mathrm{C}$ the equation is

$$
t_{90}=0.0005+0.9997333 t_{68} \text {. }
$$

\subsection{Change in Density of Water with Air Saturation}

Bignell [4] measured the change in the density of water with air saturation for 80 points in the range of 4 to $20^{\circ} \mathrm{C}$. He fitted the points to develop the equation

$$
\Delta \rho=-0.004612+0.000106 t,
$$

where $\Delta \rho$ is in $\mathrm{kg} \mathrm{m}^{-3}$. There is no need to adjust for temperature scale. Bignell concluded that "there is probably not much need to extend the work to higher temperatures because the effect diminishes and the accuracy of density metrology at these temperatures would not warrant a more accurately known correction."

\subsection{Density of Air-Saturated Water on ITS-90}

Equation (5) was added to Eq. (3) to produce an equation to be used to calculate the density, $\rho_{\text {as }}$, of air-saturated water in the temperature range 5 to $40^{\circ} \mathrm{C}$ on ITS-90:

$$
\begin{aligned}
\rho_{\text {as }} & =999.84847+6.337563 \times 10^{-2} t \\
& -8.523829 \times 10^{-3} t^{2}+6.943248 \times 10^{-5} t^{3} \\
& -3.821216 \times 10^{-7} t^{4}
\end{aligned}
$$

The uncertainty in the density of air-saturated water for an uncertainty in temperature of $1^{\circ} \mathrm{C}$ is approximately $210 \mathrm{ppm}$ or $0.21 \mathrm{~kg} \mathrm{~m}^{-3}$ at $20^{\circ} \mathrm{C}$.

\subsection{Isothermal Compressibility}

The thermal compressibility data used by Kell have been fitted against temperature on ITS-90 for the temperature range 5 to $40^{\circ} \mathrm{C}$. The resulting equation is

$$
\begin{aligned}
\kappa_{T} & =50.83101 \times 10^{-8}-3.68293 \times 10^{-9} t \\
& +7.263725 \times 10^{-11} t^{2}-6.597702 \times 10^{-13} t^{3} \\
& +2.87767 \times 10^{-15} t^{4}
\end{aligned}
$$

where $\kappa_{T}$ is thermal compressibility in $(\mathrm{kPa})^{-1}$.

The estimate of standard deviation (SD) of the residual, calculated $\kappa_{T}$-data $\kappa_{T}$, is $2.1 \times 10^{-11}$ $(\mathrm{kPa})^{-1}$; the ratio of SD to the midrange value of $\kappa_{T}$ is $4.6 \times 10^{-5}$, which is negligible for present purposes. It is not necessary to make a correction to $\kappa_{T}$ for air saturation.

The value of the isothermal compressibility of water is approximately 46.5 parts per million (ppm)/atmosphere at $20^{\circ} \mathrm{C}$. At locations where the atmospheric pressure is significantly different from 1 atmosphere $(101.325 \mathrm{kPa})$, a correction for compressibility calculated using Eq. (7) should be made. For example, at Boulder, $\mathrm{CO}$, the correction for compressibility is approximately $-8 \mathrm{ppm}$ at $20^{\circ} \mathrm{C}$.

\subsection{Compressibility-Corrected Water Density Equation}

The expression for the density of air-saturated water, $\rho_{\text {asc }}$, at an ambient pressure of $P \mathrm{kPa}$ is

$$
\rho_{\mathrm{asc}}=\rho_{\mathrm{as}}\left[1+\kappa_{T}(P-101.325)\right],
$$

where $\rho_{\mathrm{as}}$ is calculated using Eq. (6) and $\kappa_{T}$ is calculated using Eq. (7). 


\section{Tables}

Table 1 is a tabulation of values of the density of air-saturated water using Eq. (6). Table 2 is a tabulation of the values of the density of air-free water calculated using Eq. (3). Table 3 is a tabulation of values of air-free water calculated using the formulation of Wagenbreth and Blanke [1], this table has been included in this paper for purposes of comparison.

The units for water density in these tables are $\mathrm{g} / \mathrm{cm}^{3}$, as a convenience to those who routinely use these units.

Table 1. Density of air-saturated water $\left(\mathrm{g} / \mathrm{cm}^{3}\right)$ from Eq. (6) using Kell [2] data

\begin{tabular}{|c|c|c|c|c|c|c|c|c|c|c|}
\hline$t\left({ }^{\circ} \mathrm{C}\right)$ & 0.0 & 0.1 & 0.2 & 0.3 & 0.4 & 0.5 & 0.6 & 0.7 & 0.8 & 0.9 \\
\hline 5 & 0.999961 & 0.999959 & 0.999957 & 0.999955 & 0.999953 & 0.999950 & 0.999948 & 0.999945 & 0.999942 & 0.999939 \\
\hline 6 & 0.999936 & 0.999933 & 0.999930 & 0.999926 & 0.999922 & 0.999919 & 0.999915 & 0.999911 & 0.999906 & 0.999902 \\
\hline 7 & 0.999897 & 0.999893 & 0.999888 & 0.999883 & 0.999878 & 0.999872 & 0.999867 & 0.999861 & 0.999856 & 0.999850 \\
\hline 8 & 0.999844 & 0.999838 & 0.999832 & 0.999825 & 0.999819 & 0.999812 & 0.999805 & 0.999798 & 0.999791 & 0.999784 \\
\hline 9 & 0.999777 & 0.999769 & 0.999761 & 0.999754 & 0.999746 & 0.999738 & 0.999730 & 0.999721 & 0.999713 & 0.999704 \\
\hline 10 & 0.999695 & 0.999687 & 0.999678 & 0.999669 & 0.999659 & 0.999650 & 0.999640 & 0.999631 & 0.999621 & 0.999611 \\
\hline 11 & 0.999601 & 0.999591 & 0.999581 & 0.999570 & 0.999560 & 0.999549 & 0.999538 & 0.999527 & 0.999516 & 0.999505 \\
\hline 12 & 0.999494 & 0.999482 & 0.999471 & 0.999459 & 0.999447 & 0.999435 & 0.999423 & 0.999411 & 0.999398 & 0.999386 \\
\hline 13 & 0.999373 & 0.999361 & 0.999348 & 0.999335 & 0.999322 & 0.999309 & 0.999295 & 0.999282 & 0.999268 & 0.999255 \\
\hline 14 & 0.999241 & 0.999227 & 0.999213 & 0.999199 & 0.999184 & 0.999170 & 0.999156 & 0.999141 & 0.999126 & 0.999111 \\
\hline 15 & 0.999096 & 0.999081 & 0.999066 & 0.999051 & 0.999035 & 0.999019 & 0.999004 & 0.998988 & 0.998972 & 0.998956 \\
\hline 16 & 0.998940 & 0.998923 & 0.998907 & 0.998891 & 0.998874 & 0.998857 & 0.998840 & 0.998823 & 0.998806 & 0.998789 \\
\hline 17 & 0.998772 & 0.998754 & 0.998737 & 0.998719 & 0.998701 & 0.998683 & 0.998665 & 0.998647 & 0.998629 & 0.998611 \\
\hline 18 & 0.998592 & 0.998574 & 0.998555 & 0.998536 & 0.998517 & 0.998499 & 0.998479 & 0.998460 & 0.998441 & 0.998421 \\
\hline 19 & 0.998402 & 0.998382 & 0.998363 & 0.998343 & 0.998323 & 0.998303 & 0.998283 & 0.998262 & 0.998242 & 0.998221 \\
\hline 20 & 0.998201 & 0.998180 & 0.998159 & 0.998138 & 0.998117 & 0.998096 & 0.998075 & 0.998054 & 0.998032 & 0.998011 \\
\hline 21 & 0.997989 & 0.997967 & 0.997945 & 0.997924 & 0.997901 & 0.997879 & 0.997857 & 0.997835 & 0.997812 & 0.997790 \\
\hline 22 & 0.997767 & 0.997744 & 0.997721 & 0.997698 & 0.997675 & 0.997652 & 0.997629 & 0.997606 & 0.997582 & 0.997559 \\
\hline 23 & 0.997535 & 0.997511 & 0.997487 & 0.997463 & 0.997439 & 0.997415 & 0.997391 & 0.997366 & 0.997342 & 0.997317 \\
\hline 24 & 0.997293 & 0.997268 & 0.997243 & 0.997218 & 0.997193 & 0.997168 & 0.997143 & 0.997118 & 0.997092 & 0.997067 \\
\hline 25 & 0.997041 & 0.997015 & 0.996990 & 0.996964 & 0.996938 & 0.996912 & 0.996885 & 0.996859 & 0.996833 & 0.996806 \\
\hline 26 & 0.996780 & 0.996753 & 0.996726 & 0.996700 & 0.996673 & 0.996646 & 0.996619 & 0.996591 & 0.996564 & 0.996537 \\
\hline 27 & 0.996509 & 0.996482 & 0.996454 & 0.996426 & 0.996399 & 0.996371 & 0.996343 & 0.996314 & 0.996286 & 0.996258 \\
\hline 28 & 0.996230 & 0.996201 & 0.996173 & 0.996144 & 0.996115 & 0.996086 & 0.996057 & 0.996028 & 0.995999 & 0.995970 \\
\hline 29 & 0.995941 & 0.995912 & 0.995882 & 0.995853 & 0.995823 & 0.995793 & 0.995764 & 0.995734 & 0.995704 & 0.995674 \\
\hline 30 & 0.995643 & 0.995613 & 0.995583 & 0.995553 & 0.995522 & 0.995491 & 0.995461 & 0.995430 & 0.995399 & 0.995368 \\
\hline 31 & 0.995337 & 0.995306 & 0.995275 & 0.995244 & 0.995212 & 0.995181 & 0.995149 & 0.995118 & 0.995086 & 0.995054 \\
\hline 32 & 0.995023 & 0.994991 & 0.994959 & 0.994927 & 0.994894 & 0.994862 & 0.994830 & 0.994797 & 0.994765 & 0.994732 \\
\hline 33 & 0.994699 & 0.994667 & 0.994634 & 0.994601 & 0.994568 & 0.994535 & 0.994502 & 0.994468 & 0.994435 & 0.994402 \\
\hline 34 & 0.994368 & 0.994334 & 0.994301 & 0.994267 & 0.994233 & 0.994199 & 0.994165 & 0.994131 & 0.994097 & 0.994063 \\
\hline 35 & 0.994028 & 0.993994 & 0.993960 & 0.993925 & 0.993890 & 0.993856 & 0.993821 & 0.993786 & 0.993751 & 0.993716 \\
\hline 36 & 0.993681 & 0.993646 & 0.993610 & 0.993575 & 0.993539 & 0.993504 & 0.993468 & 0.993433 & 0.993397 & 0.993361 \\
\hline 37 & 0.993325 & 0.993289 & 0.993253 & 0.993217 & 0.993181 & 0.993144 & 0.993108 & 0.993071 & 0.993035 & 0.992998 \\
\hline 38 & 0.992962 & 0.992925 & 0.992888 & 0.992851 & 0.992814 & 0.992777 & 0.992740 & 0.992702 & 0.992665 & 0.992628 \\
\hline 39 & 0.992590 & 0.992553 & 0.992515 & 0.992477 & 0.992439 & 0.992401 & 0.992363 & 0.992325 & 0.992287 & 0.992249 \\
\hline
\end{tabular}


Table 2. Density of air-free water $\left(\mathrm{g} / \mathrm{cm}^{3}\right)$ from Eq. (3) using Kell [2] data

\begin{tabular}{|c|c|c|c|c|c|c|c|c|c|c|}
\hline$r\left({ }^{\circ} \mathrm{C}\right)$ & 0.0 & 0.1 & 0.2 & 0.3 & 0.4 & 0.5 & 0.6 & 0.7 & 0.8 & 0.9 \\
\hline 5 & 0.999965 & 0.999963 & 0.999961 & 0.999959 & 0.999957 & 0.999954 & 0.999952 & 0.999949 & 0.999946 & 0.999943 \\
\hline 6 & 0.999940 & 0.999937 & 0.999934 & 0.999930 & 0.999926 & 0.999923 & 0.999919 & 0.999914 & 0.999910 & 0.999906 \\
\hline 7 & 0.999901 & 0.999896 & 0.999892 & 0.999887 & 0.999881 & 0.999876 & 0.999871 & 0.999865 & 0.999860 & 0.999854 \\
\hline 8 & 0.999848 & 0.999842 & 0.999835 & 0.999829 & 0.999822 & 0.999816 & 0.999809 & 0.999802 & 0.999795 & 0.999788 \\
\hline 9 & 0.999780 & 0.999773 & 0.999765 & 0.999757 & 0.999749 & 0.999741 & 0.999733 & 0.999725 & 0.999716 & 0.999708 \\
\hline 10 & 0.999699 & 0.999690 & 0.999681 & 0.999672 & 0.999663 & 0.999653 & 0.999644 & 0.999634 & 0.999624 & 0.999615 \\
\hline 11 & 0.999604 & 0.999594 & 0.999584 & 0.999574 & 0.999563 & 0.999552 & 0.999541 & 0.999531 & 0.999519 & 0.999508 \\
\hline 12 & 0.999497 & 0.999485 & 0.999474 & 0.999462 & 0.999450 & 0.999438 & 0.999426 & 0.999414 & 0.999402 & 0.999389 \\
\hline 13 & 0.999377 & 0.999364 & 0.999351 & 0.999338 & 0.999325 & 0.999312 & 0.999299 & 0.999285 & 0.999272 & 0.999258 \\
\hline 14 & 0.999244 & 0.999230 & 0.999216 & 0.999202 & 0.999188 & 0.999173 & 0.999159 & 0.999144 & 0.999129 & 0.999114 \\
\hline 15 & 0.999099 & 0.999084 & 0.999069 & 0.999053 & 0.999038 & 0.999022 & 0.9990007 & 0.998991 & 0.998975 & 0.998959 \\
\hline 16 & 0.998943 & 0.998926 & 0.998910 & 0.998893 & 0.998877 & 0.998860 & 0.998843 & 0.998826 & 0.998809 & 0.998792 \\
\hline 17 & 0.998774 & 0.998757 & 0.998739 & 0.998722 & 0.998704 & 0.998686 & 0.998668 & 0.998650 & 0.998632 & 0.998613 \\
\hline 18 & 0.998595 & 0.998576 & 0.998558 & 0.998539 & 0.998520 & 0.998501 & 0.998482 & 0.998463 & 0.998444 & 0.998424 \\
\hline 19 & 0.998405 & 0.998385 & 0.998365 & 0.998345 & 0.998325 & 0.998305 & 0.998285 & 0.998265 & 0.998244 & 0.998224 \\
\hline 20 & 0.998203 & 0.998183 & 0.998162 & 0.998141 & 0.998120 & 0.998099 & 0.998077 & 0.998056 & 0.998035 & 0.998013 \\
\hline 21 & 0.997991 & 0.997970 & 0.997948 & 0.997926 & 0.997904 & 0.997882 & 0.997859 & 0.997837 & 0.997815 & 0.997792 \\
\hline 22 & 0.997769 & 0.997746 & 0.997724 & 0.997701 & 0.997678 & 0.997654 & 0.997631 & 0.997608 & 0.997584 & 0.997561 \\
\hline 23 & 0.997537 & 0.997513 & 0.997489 & 0.997465 & 0.997441 & 0.997417 & 0.997393 & 0.997369 & 0.997344 & 0.997320 \\
\hline 24 & 0.997295 & 0.997270 & 0.997245 & 0.997220 & 0.997195 & 0.997170 & 0.997145 & 0.997120 & 0.997094 & 0.997069 \\
\hline 25 & 0.997043 & 0.997017 & 0.996992 & 0.996966 & 0.996940 & 0.996914 & 0.996887 & 0.996861 & 0.996835 & 0.996808 \\
\hline 26 & 0.996782 & 0.996755 & 0.996728 & 0.996701 & 0.996675 & 0.996648 & 0.996620 & 0.996593 & 0.996566 & 0.996539 \\
\hline 27 & 0.996511 & 0.996483 & 0.996456 & 0.996428 & 0.996400 & 0.996372 & 0.996344 & 0.996316 & 0.996288 & 0.996260 \\
\hline 28 & 0.996231 & 0.996203 & 0.996174 & 0.996146 & 0.996117 & 0.996088 & 0.996059 & 0.996030 & 0.996001 & 0.995972 \\
\hline 29 & 0.995942 & 0.995913 & 0.995884 & 0.995854 & 0.995824 & 0.995795 & 0.995765 & 0.995735 & 0.995705 & 0.995675 \\
\hline 30 & 0.995645 & 0.995615 & 0.995584 & 0.995554 & 0.995523 & 0.995493 & 0.995462 & 0.995431 & 0.995401 & 0.995370 \\
\hline 31 & 0.995339 & 0.995307 & 0.995276 & 0.995245 & 0.995214 & 0.995182 & 0.995151 & 0.995119 & 0.995087 & 0.995056 \\
\hline 32 & 0.995024 & 0.994992 & 0.994960 & 0.994928 & 0.994895 & 0.994863 & 0.994831 & 0.994798 & 0.994766 & 0.994733 \\
\hline 33 & 0.994701 & 0.994668 & 0.994635 & 0.994602 & 0.994569 & 0.994536 & 0.994503 & 0.994469 & 0.994436 & 0.994403 \\
\hline 34 & 0.994369 & 0.994335 & 0.994302 & 0.994268 & 0.994234 & 0.994200 & 0.994166 & 0.994132 & 0.994098 & 0.994064 \\
\hline 35 & 0.994029 & 0.993995 & 0.993960 & 0.993926 & 0.993891 & 0.993856 & 0.993822 & 0.993787 & 0.993752 & 0.993717 \\
\hline 36 & 0.993682 & 0.993646 & 0.993611 & 0.993576 & 0.993540 & 0.993505 & 0.993469 & 0.993433 & 0.993398 & 0.993362 \\
\hline 37 & 0.993326 & 0.993290 & 0.993254 & 0.993217 & 0.993181 & 0.993145 & 0.993108 & 0.993072 & 0.993035 & 0.992999 \\
\hline 38 & 0.992962 & 0.992925 & 0.992888 & 0.992851 & 0.992814 & 0.992777 & 0.992740 & 0.992703 & 0.992665 & 0.992628 \\
\hline 39 & 0.992590 & 0.992553 & 0.992515 & 0.992478 & 0.992440 & 0.992402 & 0.992364 & 0.992326 & 0.992288 & 0.992249 \\
\hline
\end{tabular}


Table 3. Density of air-free water $\left(\mathrm{g} / \mathrm{cm}^{3}\right)$ from formulation of Wagenbreth and Blanke [1]

\begin{tabular}{|c|c|c|c|c|c|c|c|c|c|c|}
\hline$t\left({ }^{\circ} \mathrm{C}\right)$ & 0.0 & 0.1 & 0.2 & 0.3 & 0.4 & 0.5 & 0.6 & 0.7 & 0.8 & 0.9 \\
\hline 5 & 0.999964 & 0.999962 & 0.999960 & 0.999958 & 0.999956 & 0.999954 & 0.999951 & 0.999949 & 0.999946 & 0.999943 \\
\hline 6 & 0.999940 & 0.999937 & 0.999933 & 0.999930 & 0.999926 & 0.999922 & 0.999918 & 0.999914 & 0.999910 & 0.999906 \\
\hline 7 & 0.999901 & 0.999896 & 0.999892 & 0.999887 & 0.999881 & 0.999876 & 0.999871 & 0.999865 & 0.999860 & 0.999854 \\
\hline 8 & 0.999848 & 0.999842 & 0.999835 & 0.999829 & 0.999822 & 0.999816 & 0.999809 & 0.999802 & 0.999795 & 0.999787 \\
\hline 9 & 0.999780 & 0.999773 & 0.999765 & 0.999757 & 0.999749 & 0.999741 & 0.999733 & 0.999725 & 0.999716 & 0.999707 \\
\hline 10 & 0.999699 & 0.999690 & 0.999681 & 0.999672 & 0.999662 & 0.999653 & 0.999643 & 0.999634 & 0.999624 & 0.999614 \\
\hline 11 & 0.999604 & 0.999594 & 0.999583 & 0.999573 & 0.999562 & 0.999552 & 0.999541 & 0.999530 & 0.999519 & 0.999507 \\
\hline 12 & 0.999496 & 0.999485 & 0.999473 & 0.999461 & 0.999449 & 0.999437 & 0.999425 & 0.999413 & 0.999401 & 0.999388 \\
\hline 13 & 0.999376 & 0.999363 & 0.999350 & 0.999337 & 0.999324 & 0.999311 & 0.999297 & 0.999284 & 0.999270 & 0.999256 \\
\hline 14 & 0.999243 & 0.999229 & 0.999215 & 0.999200 & 0.999186 & 0.999172 & 0.999157 & 0.999142 & 0.999128 & 0.999113 \\
\hline 15 & 0.999098 & 0.999083 & 0.999067 & 0.999052 & 0.999036 & 0.999021 & 0.999005 & 0.998989 & 0.998973 & 0.998957 \\
\hline 16 & 0.998941 & 0.998925 & 0.998908 & 0.998892 & 0.998875 & 0.998858 & 0.998841 & 0.998824 & 0.998807 & 0.998790 \\
\hline 17 & 0.998773 & 0.998755 & 0.998738 & 0.998720 & 0.998702 & 0.998684 & 0.998666 & 0.998648 & 0.998630 & 0.998612 \\
\hline 18 & 0.998593 & 0.998575 & 0.998556 & 0.998537 & 0.998519 & 0.998500 & 0.998480 & 0.998461 & 0.998442 & 0.998422 \\
\hline 19 & 0.998403 & 0.998383 & 0.998364 & 0.998344 & 0.998324 & 0.998304 & 0.998284 & 0.998263 & 0.998243 & 0.998222 \\
\hline 20 & 0.998202 & 0.998181 & 0.998160 & 0.998139 & 0.998118 & 0.998097 & 0.998076 & 0.998055 & 0.998033 & 0.998012 \\
\hline 21 & 0.997990 & 0.997968 & 0.997947 & 0.997925 & 0.997903 & 0.997881 & 0.997858 & 0.997836 & 0.997814 & 0.997791 \\
\hline 22 & 0.997768 & 0.997746 & 0.997723 & 0.997700 & 0.997677 & 0.997654 & 0.997630 & 0.997607 & 0.997584 & 0.997560 \\
\hline 23 & 0.997536 & 0.997513 & 0.997489 & 0.997465 & 0.997441 & 0.997417 & 0.997392 & 0.997368 & 0.997344 & 0.997319 \\
\hline 24 & 0.997294 & 0.997270 & 0.997245 & 0.997220 & 0.997195 & 0.997170 & 0.997145 & 0.997119 & 0.997094 & 0.997068 \\
\hline 25 & 0.997043 & 0.997017 & 0.996991 & 0.996966 & 0.996940 & 0.996913 & 0.996887 & 0.996861 & 0.996835 & 0.996808 \\
\hline 26 & 0.996782 & 0.996755 & 0.996723 & 0.996702 & 0.996675 & 0.996648 & 0.996621 & 0.996593 & 0.996566 & 0.996539 \\
\hline 27 & 0.996511 & 0.996484 & 0.996456 & 0.996428 & 0.996401 & 0.996373 & 0.996345 & 0.996316 & 0.996288 & 0.996260 \\
\hline 28 & 0.996232 & 0.996203 & 0.996175 & 0.996146 & 0.996117 & 0.996088 & 0.996060 & 0.996031 & 0.996001 & 0.995972 \\
\hline 29 & 0.996943 & 0.995914 & 0.995884 & 0.995855 & 0.995825 & 0.995795 & 0.995765 & 0.995736 & 0.995706 & 0.995676 \\
\hline 30 & 0.995645 & 0.995615 & 0.995585 & 0.995554 & 0.995524 & 0.995493 & 0.995463 & 0.995432 & 0.995401 & 0.995370 \\
\hline 31 & 0.995339 & 0.995308 & 0.995277 & 0.995246 & 0.995214 & 0.995183 & 0.995151 & 0.995120 & 0.995088 & 0.995056 \\
\hline 32 & 0.995024 & 0.994992 & 0.994960 & 0.994928 & 0.994896 & 0.994864 & 0.994831 & 0.994799 & 0.994766 & 0.994734 \\
\hline 33 & 0.994701 & 0.994668 & 0.994635 & 0.994602 & 0.994569 & 0.994536 & 0.994503 & 0.994470 & 0.994436 & 0.994403 \\
\hline 34 & 0.994369 & 0.994336 & 0.994302 & 0.994268 & 0.994234 & 0.994201 & 0.994167 & 0.994132 & 0.994098 & 0.994064 \\
\hline 35 & 0.994030 & 0.993995 & 0.993961 & 0.993926 & 0.993891 & 0.993857 & 0.993822 & 0.993787 & 0.993752 & 0.993717 \\
\hline 36 & 0.993682 & 0.993647 & 0.993611 & 0.993576 & 0.993541 & 0.993505 & 0.993469 & 0.993434 & 0.993398 & 0.993362 \\
\hline 37 & 0.993326 & 0.993290 & 0.993254 & 0.993218 & 0.993182 & 0.993146 & 0.993109 & 0.993073 & 0.993036 & 0.993000 \\
\hline 38 & 0.992963 & 0.992926 & 0.992889 & 0.992852 & 0.992815 & 0.992778 & 0.992741 & 0.992704 & 0.992667 & 0.992629 \\
\hline 39 & 0.992592 & 0.992554 & 0.992517 & 0.992479 & 0.992442 & 0.992404 & 0.992366 & 0.992328 & 0.992290 & 0.992252 \\
\hline
\end{tabular}




\section{Summary}

Equation (3) can be used to calculate the density of air-free water in the temperature range of 5 to $40^{\circ} \mathrm{C}$ in ITS-90 at one atmosphere.

Equation (6) can be used to calculate the density of air-saturated water in the same temperature range at one atmosphere.

Equation (8) can be used to calculate the density of air-saturated water in the same temperature range at an ambient pressure of $P \mathrm{kPa}$.

The use of Eq. (6) for air-saturated water, and Eq. (8) where appropriate, is recommended for calculation of water density.

\section{References}

[1] H. Wagenbreth, and W. Blanke, PTB Mitt. 81, 412 (1971).

[2] G. S. Kell, J. Chem. Eng. Data 20, 97 (1975).

[3] B. W. Mangum, and G. T. Furukawa, Natl. Inst. Stand. Technol. Technical Note 1265 (1990).

[4] N. Bignell, Metrologia 19, 57 (1983).

About the authors: Frank E. Jones is a physicist and an independent consultant who retired from the National Bureau of Standards (now NIST) in 1987. Georgia L. Harris is a physical scientist with the Office of Weights and Measures at NIST. The National Institute of Standards and Technology is an agency of the Technology Administration, U.S. Department of Commerce. 